Vietnam Journal of Mechanics, VAST, Vol.41, No. 2 (2019), pp. $141-155$

DOI: https://doi.org/10.15625/0866-7136/13073

\title{
KINEMATIC AND DYNAMIC ANALYSIS OF A SERIAL MANIPULATOR WITH LOCAL CLOSED LOOP MECHANISMS
}

\author{
Chu Anh My*, Vu Minh Hoan \\ Le Quy Don University of Technology, Hanoi, Vietnam \\ *E-mail: mychuanh@yahoo.com \\ Received: 05 September 2018 / Published online: 01 April 2019
}

\begin{abstract}
This paper addresses the kinematic and dynamic modelling and analysis for a robot arm consisting of two hydraulic cylinders driving two revolute joints of the arm. The two cylinders and relevant links of the robot constitute two local closed kinematic chains added to the main robot mechanism. Therefore, the number of the generalized coordinates of the mechanical system is increased, and the mathematical modelling is more complex that requires a formulation of constraint equations with respect to the local closed chains. By using the Lagrangian formulation with Lagrangian Multipliers, the dynamic equations are first derived with respect to all extended generalized coordinates. Then a compact form of the dynamic equations is yielded by canceling the Multipliers. Since the obtained dynamic equations are expressed in terms of independent generalized coordinates which are selected according to active joint variables of the arm, the equations could be best suitable for control law design and implementation. The simulation of the forward and inverse kinematics and dynamics of the arm demonstrates the motion behavior of the robot system.
\end{abstract}

Keywords: hydraulic robot; robot kinematics; robot dynamics; local closed mechanism.

\section{INTRODUCTION}

Most of industrial manipulators commonly used in industries are usually actuated by electric motors, such as the welding robots, the assembly robots, etc. The use of electric motors actuating active robot joints possesses several advantages: easy to control, high positioning accuracy, and high flexibility. However, if a manipulator is designed to operate in a large workspace with high loading capability, the use of electric motors for the design could lead to a very heavy architecture of the robot. Counterweights could be added to balance to shaking forces. In that case, hydraulic cylinders driving robot joints is often used for the design. The presence of hydraulic cylinders increases the stiffness of robot structure so that the robot is capable of handling heavy parts in a larger operational space. Moreover, the counterweights could be avoided since the cylinders actuating revolute joints play a role of auxiliary links appended to the main structure.

(C) 2019 Vietnam Academy of Science and Technology 
Though there will be advantages when using hydraulic cylinders for robot designs, the presence of cylinders in a robot architecture involves complex procedures for the mathematical modelling, analysis and control. The addition of cylinders to the conventional serial kinematic chain of robot arm architecture could constitute local closed kinematic chains within the entire robot mechanism. Issues related to the hybrid serial-parallel feature of the robot structure, the geometry, the mass and the inertia of cylinders must be taken into account.

In the literature, numerous works have been carried out to investigate several aspects related to the dynamic modeling and analysis of serial manipulators and parallel robots [1-21]. The fundamentals of kinematic and dynamic modelling and analysis of serial manipulators can be found in [1,2], where Denavit-Hartenberg approach was mostly used for the kinematic modelling and D'Alembert-Lagrange Formulation for the dynamic modelling. As for more complex robotic systems, there has been a number of researches dealing with different issues related to the kinematics and the dynamics as well. The research presented in [3] studied the kinematic and dynamic modelling for closed chain manipulators, [4] addressed algorithms for the dynamic analysis of serial robots having a large number of joints, [5] investigated the inverse kinematics and dynamics of the redundant robots, [6] studied the dynamics of mobile serial manipulators. In parallel with researches concerning with the serial robot dynamics, a massive number of researches related to the dynamics and control of parallel robots has been addressed as well such as publications [7-21]. The researches $[8,10,13]$ investigated methods for the inverse and forward dynamic modelling and analysis of the 3-PRS type parallel manipulators. The Screw theory was used in [9], and the matrix approach was employed in [11] for the dynamics and control of the parallel robots. A general solution to the problem of dynamic modelling and analysis of parallel robots was presented in [12]. For the issue of control law design and development, [14] investigated a model-based technique, [15-17] addressed the robust control algorithms, whereas the sliding mode control was designed in [18]. The earlier foundation for control law designed for the parallel robots one can find in [19], in which the computed torque technique was used as for those of the common serial manipulators. The control law design for a 6-DOF hexapod robot was presented in [20], and a technique to improve the control quality for parallel robots was investigated in [21].

In recent years, there have been several attempts to investigate the dynamic modelling and analysis of the hybrid serial-parallel robots [22-31]. Ibrahim \& Khalil [22] studied recursive solutions for obtaining the inverse and direct dynamic models of hybrid robots that are constructed by serially connected non-redundant parallel modules. Pisla et al. [23] addressed geometric and kinematic models of a surgical hybrid robot used for camera and active instruments positioning. Tanev [24] investigated a kinematic analysis of a hybrid parallel-serial robot manipulator which consists of two serially connected parallel mechanisms. Each mechanism has three degrees of freedom. Xu et al. [25] presented a particular hybrid manipulator for computer controlled ultra-precision freeform polishing. This hybrid manipulator is composed of a 3-DOF parallel module, a 2-DOF serial module and a turntable providing a redundant DOF. Zeng \& Fang [26] studied a method of structural synthesis and analysis of serial-parallel hybrid mechanisms with 
spatial multi-loop kinematic chains based on the displacement group theory. Zeng \& Ehmann [27] presented a design method for parallel hybrid-loop manipulators, which is based on the constrained motion properties of related spatial over constrained linkages and general parallel mechanisms. Zhang \& Gao [28] investigated a method for performance optimization of a 5-DOF compliant hybrid parallel robot micromanipulator. The issues related to kinematics modelling and analysis for the robots were also addressed in [29-31].

Though there has been a number of researches dealing with the dynamic modelling and analysis of different robot architectures, little attention has been paid to robots actuated by hydraulic actuators. Issues related to the mathematical modelling of manipulators which have local closed kinematic chains constituted by adding hydraulic cylinders seem to be overlooked.

In this paper, the dynamic modelling and analysis of such a robot arm having two hydraulic cylinders constituting two local closed chains appended to a conventional arm mechanism are addressed. First, the kinematic equations and constraints equations are derived, by using the notation of Denavit-Hartenberg. Second, Lagrange's formulation is used to establish differential equations of motion, with the use of Lagrangian multipliers. Finally, based on the formulated DAEs, a technique of Lagrangian multipliers cancellation is employed to obtain ODEs describing the dynamics of the entire robot system. Finally, the dynamic analysis is carried out with the help of the symbolic and numeric computing Maple and Matlab softwares. As a result, the proposed investigation could be used further for the purposes of dynamic simulation and control law design for a class of hydraulic architecture robots.

\section{KINEMATICS}

Let's consider a robot in Fig 1. Different from the conventional robot arm architecture, the considering manipulator has two hydraulic cylinders. The first cylinder AC is to drive the revolute joint $B$ (joint variable $\theta_{2}$ ), and the second one EG drives the joint $\mathrm{F}$ (joint variable $\theta_{5}$ ). Due to the use of the cylinders, two closed kinematic chains are constituted: $\mathrm{ABCD}$ and EFGH. The robot have three DOFs and three active joints. The first active joint is a revolute joint (joint 1 ) with joint variable $\theta_{1}$. The second and the last active joints are prismatic joints (with the joint variables $d_{4}$ and $d_{7}$ ) because of the relative motion of the two parts of the hydraulic cylinders. Therefore, among eight joint variables $\theta_{1}, d_{4}, d_{7}, \theta_{2}, \theta_{3}, \theta_{4}, \theta_{5}$ and $\theta_{6}$ (see Fig. 1), only three joint variables $\theta_{1}, d_{4}$ and $d_{7}$ are independent.

In Fig. 1, Oxyz is defined as a reference frame, and $O_{E} x_{E} y_{E} z_{E}$ as an end effector frame, of which the origin locates at point E. Other local coordinate systems attached to links are defined by using Denavit-Hartenberg (DH) notations. $l_{1}, l_{10}, l_{11}, l_{2}, l_{21}, l_{22}, l_{23}$, $l_{3}, l_{5}, l_{51}, l_{6}$ are geometric parameters of links. The main mechanism of the arm includes three links: link 1, link 2, and link 5. The first local closed chain (ABCDA) involves link 1 , link 2, link 3 and link 4 . The second local closed chain (KFGHK) relates to link 2, link 5, link 6 and link 7. Based on all the coordinates in Fig. 1 which are defined with respect to the $\mathrm{DH}$ convention, the parameters of the robot links are presented in the following tables. 


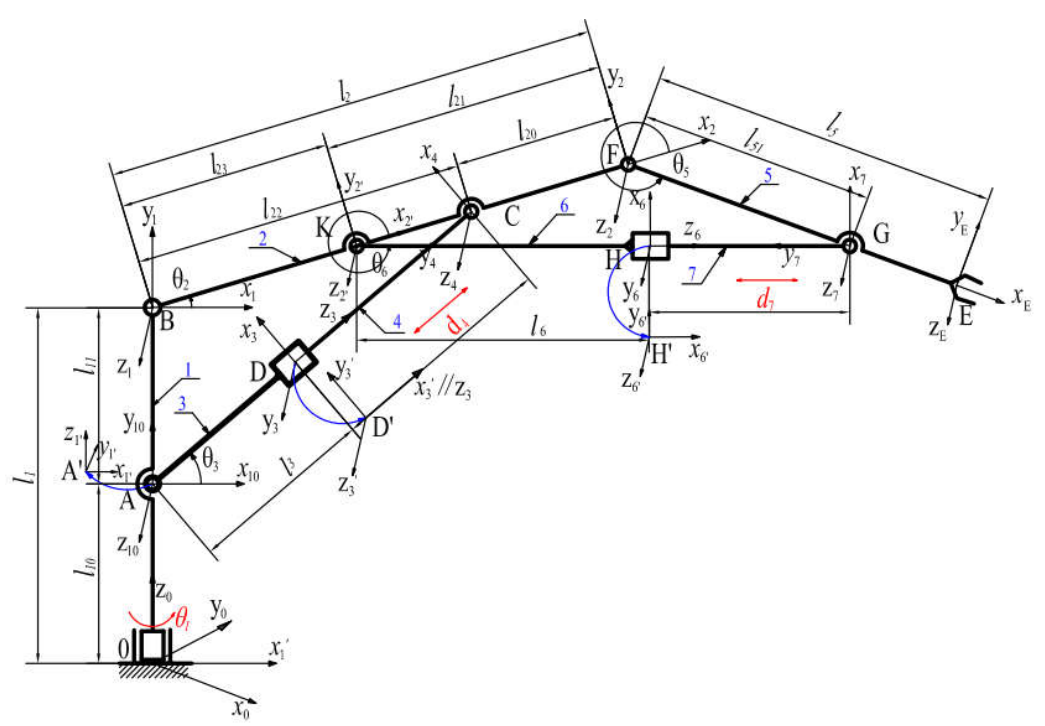

Fig. 1. A hybrid serial-parallel robot arm

Table 1. The DH parameters related to the main mechanism

\begin{tabular}{ccccc}
\hline$i$ & $\theta_{i}$ & $d_{i}$ & $a_{i}$ & $\alpha_{i}$ \\
\hline 1 & $\theta_{1}$ & $l_{1}$ & 0 & $\pi / 2$ \\
2 & $\theta_{2}$ & 0 & $l_{2}$ & 0 \\
5 & $\theta_{5}$ & 0 & $l_{5}$ & 0 \\
\hline
\end{tabular}

Table 2. The DH parameters related to the main mechanism

\begin{tabular}{cccccccccc}
\hline$i$ & $\theta_{i}$ & $d_{i}$ & $a_{i}$ & $\alpha_{i}$ & $i$ & $\theta_{i}$ & $d_{i}$ & $a_{i}$ & $\alpha_{i}$ \\
\hline $3^{\prime}$ & $\theta_{3}$ & 0 & $l_{3}$ & 0 & $1^{\prime}$ & 0 & 0 & 0 & $-\pi / 2$ \\
3 & $\pi / 2$ & 0 & 0 & $\pi / 2$ & 1 & 0 & $l_{11}$ & 0 & $\pi / 2$ \\
4 & 0 & $d_{4}$ & 0 & $-\pi / 2$ & 2 & $\theta_{2}$ & 0 & $l_{22}$ & 0 \\
\hline
\end{tabular}

Table 3. The DH parameters related the local closed chain KFGHK

\begin{tabular}{cccccccccc}
\hline$i$ & $\theta_{i}$ & $d_{i}$ & $a_{i}$ & $\alpha_{i}$ & $i$ & $\theta_{i}$ & $d_{i}$ & $a_{i}$ & $\alpha_{i}$ \\
\hline $6^{\prime}$ & $\theta_{6}$ & 0 & $l_{6}$ & 0 & 2 & 0 & 0 & $l_{21}$ & 0 \\
6 & $\pi / 2$ & 0 & 0 & $\pi / 2$ & 5 & $\theta_{5}$ & 0 & $l_{51}$ & 0 \\
7 & 0 & $d_{7}$ & 0 & $-\pi / 2$ & & & & & \\
\hline
\end{tabular}

Based on the parameters in Tabs. 1-3, the transformation matrices describing the motion of any two successive coordinate systems can be derived accordingly. For the main mechanism, the cumulative matrix $\mathbf{H}_{0 E}$ is computed as follows

$\mathbf{H}_{0 E}=\left[\begin{array}{cccc}\cos \theta_{1} \cos \left(\theta_{2}+\theta_{5}\right) & -\cos \theta_{1} \sin \left(\theta_{2}+\theta_{5}\right) & \sin \theta_{1} & -\cos \theta_{1}\left(l_{5} \sin \theta_{2} \sin \theta_{5}-l_{5} \cos \theta_{5} \cos \theta_{2}-l_{2} \cos \theta_{2}\right) \\ \sin \theta_{1} \cos \left(\theta_{2}+\theta_{5}\right) & -\sin \theta_{1} \sin \left(\theta_{2}+\theta_{5}\right) & -\cos \theta_{1} & -\sin \theta_{1}\left(l_{5} \sin \theta_{2} \sin \theta_{5}-l_{5} \cos \theta_{5} \cos \theta_{2}-l_{2} \cos \theta_{2}\right) \\ \sin \left(\theta_{2}+\theta_{5}\right) & \cos \left(\theta_{2}+\theta_{5}\right) & 0 & l_{5} \sin \theta_{2} \cos \theta_{5}+l_{5} \cos \theta_{2} \sin \theta_{5}+l_{2} \sin \theta_{2}+l_{1} \\ 0 & 0 & 0 & 1\end{array}\right]$. 
The matrix $\mathbf{H}_{0 E}$ describes the kinematics of motion of one active joint and two passive joints. In other words, $\mathbf{H}_{O E}$ characterizes the kinematic relationship of the end link of the arm with the use of only one independent joint variable $\theta_{1}$ and other two dependent joint variables $\theta_{2}$ and $\theta_{5}$.

In order to determine the kinematic relationship with all independent joint variables, the constraints raised due to the local closed chains need to be taken into account.

\subsection{Constraint equations}

Consider the first local closed chain (ABCDA). In order to write constraint equations regarding of this closed feature of the mechanism, a virtual cut at the joint $C$ is made, so that transformation matrices written with respect to two routines $\mathrm{ABC}$ and ADC, respectively, are yielded as

$$
\begin{gathered}
\mathbf{H}_{A B C}=\left[\begin{array}{cccc}
\cos \theta_{2} & -\sin \theta_{2} & 0 & l_{22} \cos \theta_{2} \\
\sin \theta_{2} & \cos \theta_{2} & 0 & l_{22} \sin \theta_{2}+l_{11} \\
0 & 0 & 1 & 0 \\
0 & 0 & 0 & 1
\end{array}\right], \\
\mathbf{H}_{A D C}=\left[\begin{array}{cccc}
-\sin \theta_{3} & -\cos \theta_{3} & 0 & d_{4} \cos \theta_{3}+l_{3} \cos \theta_{3} \\
\cos \theta_{3} & -\sin \theta_{3} & 0 & d_{4} \sin \theta_{3}+l_{3} \sin \theta_{3} \\
0 & 0 & 1 & 0 \\
0 & 0 & 0 & 1
\end{array}\right] .
\end{gathered}
$$

In the first routine, the position $\mathbf{C}^{1}$ of the point $C$ is $\mathbf{C}^{1}=\left[\begin{array}{c}l_{22} \cos \theta_{2} \\ l_{22} \sin \theta_{2}+l_{11} \\ 0\end{array}\right]$, and in the second one, the point $C$ is calculated as $\mathbf{C}^{2}=\left[\begin{array}{c}d_{4} \cos \theta_{3}+l_{3} \cos \theta_{3} \\ d_{4} \sin \theta_{3}+l_{3} \sin \theta_{3} \\ 0\end{array}\right]$. Therefore, two independent constraint equations involved in this closed chain are determined as

$$
\begin{aligned}
& f_{1}=l_{22} \cos \theta_{2}-\left(d_{4}+l_{3}\right) \cos \theta_{3}=0, \\
& f_{2}=l_{22} \sin \theta_{2}-\left(d_{4}+l_{3}\right) \sin \theta_{3}+l_{11}=0, \\
& \sin \left(\theta_{3}\right)=\frac{\left(l_{3}+d_{4}\right)^{2}+\left(l_{11}\right)^{2}-\left(l_{22}\right)^{2}}{2\left(l_{3}+d_{4}\right) l_{11}}, \\
& \sin \left(\theta_{2}\right)=\frac{\left(l_{3}+d_{4}\right)^{2}-l_{11}^{2}-l_{22}^{2}}{2 l_{11} l_{22}} .
\end{aligned}
$$

Similarly, the other two constraint equations involved in the second local closed chain KFGHK are obtained as

$$
\begin{aligned}
& f_{3}=l_{51} \cos \theta_{5}+l_{21}-d_{7} \cos \theta_{6}-l_{6} \cos \theta_{6}=0, \\
& f_{4}=l_{51} \sin \theta_{5}-d_{7} \sin \theta_{6}-l_{6} \sin \theta_{6}=0,
\end{aligned}
$$




$$
\begin{aligned}
& \cos \left(\theta_{5}\right)=\frac{\left(l_{6}+d_{7}\right)^{2}-\left(l_{51}\right)^{2}-\left(l_{21}\right)^{2}}{2\left(l_{51}\right)\left(l_{21}\right)}, \\
& \cos \left(\theta_{6}\right)=\frac{\left(l_{6}+d_{7}\right)^{2}-\left(l_{51}\right)^{2}+\left(l_{21}\right)^{2}}{2\left(l_{21}\right)\left(l_{6}+d_{7}\right)} .
\end{aligned}
$$

\subsection{Forward kinematics simulation}

Based on Eqs. (1), (5) and (7), with the inputs given as $\theta_{1}(t)=\frac{\pi}{18} \times t, d_{4}(t)=0.1+$ $0.005 \times t$ and $d_{7}(t)=0.1+0.02 \times t$, the position of the end effector $\mathbf{E}=\left[x_{E}(t) y_{E}(t) z_{E}(t)\right]^{T}$ and the velocity $\dot{\mathbf{E}}=\left[\dot{x}_{E}(t) \dot{y}_{E}(t) \dot{z}_{E}(t)\right]^{T}$ can be numerically computed and shown in the following Figs. 2, 3 and 4 , respectively .

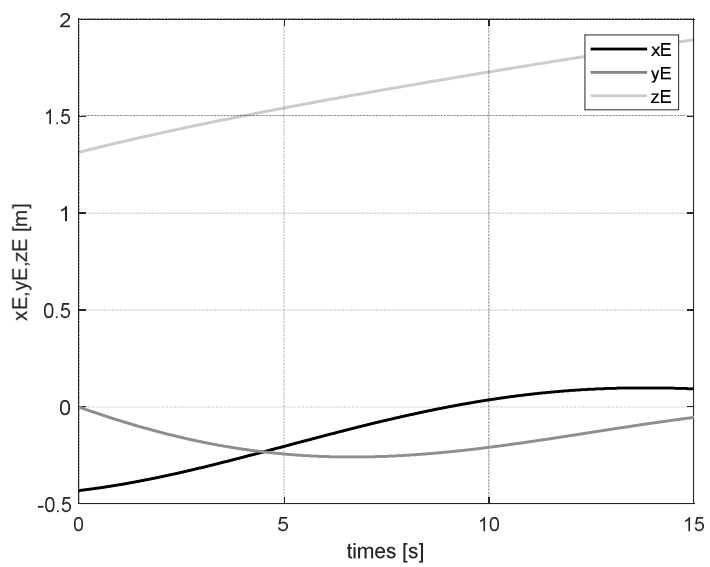

Fig. 2. $\mathbf{E}=\left[x_{E}(t) y_{E}(t) z_{E}(t)\right]^{T}$

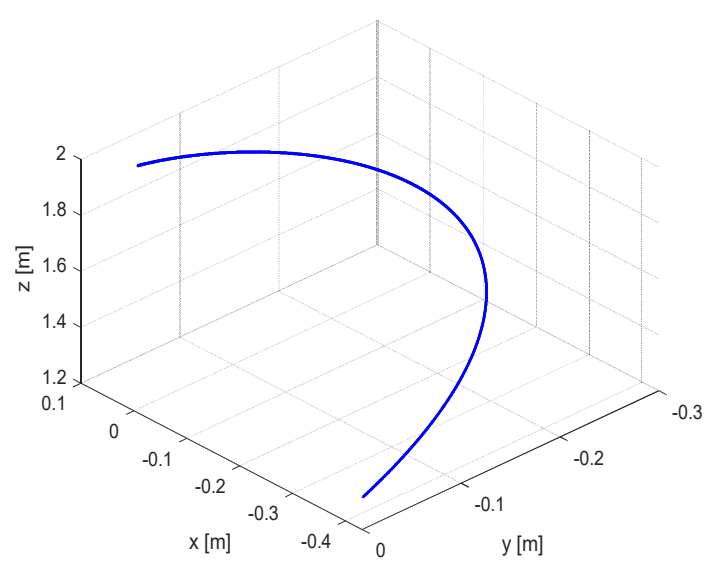

Fig. 3. A trajectory of $\mathbf{E}$ in the workspace

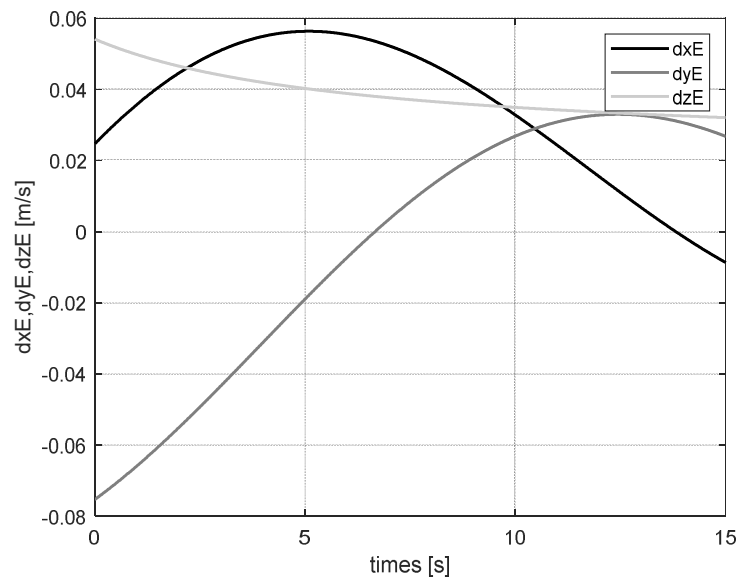

Fig. 4. $\dot{\mathbf{E}}=\left[\dot{x}_{E}(t) \dot{y}_{E}(t) \dot{z}_{E}(t)\right]^{T}$ 


\subsection{Inverse kinematics simulation}

Given a trajectory of the end-effector in the work space, $\mathbf{E}=\left[x_{E}(t) y_{E}(t) z_{E}(t)\right]^{T}$, the joint variables are calculated as follows

$$
\begin{gathered}
\theta_{1}=\arcsin \left(A /\left(1+A^{2}\right)^{1 / 2}\right) \\
d_{4}=\sqrt{2 l_{11} l_{22} \sin \theta_{2}+l_{11}^{2}+l_{22}^{2}}-l_{3} \\
d_{7}=\sqrt{2 l_{51} l_{21} \cos \theta_{5}+l_{51}^{2}+l_{21}^{2}}-l_{6} \\
\cos \left(\theta_{5}\right)=\frac{y_{M}^{2}}{2 l_{2} l_{5} B^{2}}+\frac{\left(z_{M}-l_{1}\right)^{2}-l_{2}^{2}-l_{5}^{2}}{2 l_{2} l_{5}}=C \\
\sin \left(\theta_{2}\right)=\frac{m n \pm \sqrt{\Delta^{\prime}}}{\left(n^{2}+p^{2}\right)} ; \Delta^{\prime}=m^{2} n^{2}-\left(n^{2}+p^{2}\right)\left(m^{2}-p^{2}\right)
\end{gathered}
$$

where $A=\frac{y_{E}}{x_{E}} ; B=\sqrt{\frac{A^{2}}{1+A^{2}}} ; m=\left(z_{E}-l_{1}\right) ; n=\left(l_{5} C+l_{2}\right) ; p=l_{5} \sqrt{1-C^{2}}$.

Figs. 5 and 6 show the values of $\theta_{1}(t), d_{4}(t), d_{7}(t), \theta_{2}(t)$ and $\theta_{5}(t)$ computed with respect to a trajectory given as

$$
\begin{aligned}
& x_{E}=\frac{1}{8000}\left(\frac{1}{2}+\left(\frac{t^{2}}{1600}+\frac{t}{32}-\frac{55}{64}\right)\right) \cos \left(\frac{\pi t}{18}\right) \sqrt{92160000-\left((t+100)^{2}-10000\right)^{2}}-\frac{t(t+200)}{12800000} \cos \left(\frac{\pi t}{18}\right) \sqrt{2560000-\left((t+25)^{2}-2000\right)^{2}}, \\
& y_{E}=\frac{1}{8000}\left(\frac{1}{2}+\left(\frac{t^{2}}{1600}+\frac{t}{32}-\frac{55}{64}\right)\right) \sin \left(\frac{\pi t}{18}\right) \sqrt{92160000-\left((t+100)^{2}-10000\right)^{2}}-\frac{t(t+200)}{12800000} \sin \left(\frac{\pi t}{18}\right) \sqrt{2560000-\left((t+25)^{2}-2000\right)^{2}} \\
& z_{E}=\frac{1}{12800000} \sqrt{2560000-\left((t+25)^{2}-2000\right)^{2}} \sqrt{92160000-\left((t+100)^{2}-10000\right)^{2}}+\frac{t^{4}}{12800000}+\frac{t^{3}}{51200}+\frac{377 t^{2}}{512000}-\frac{23 t}{2560}+\frac{7}{10} .
\end{aligned}
$$

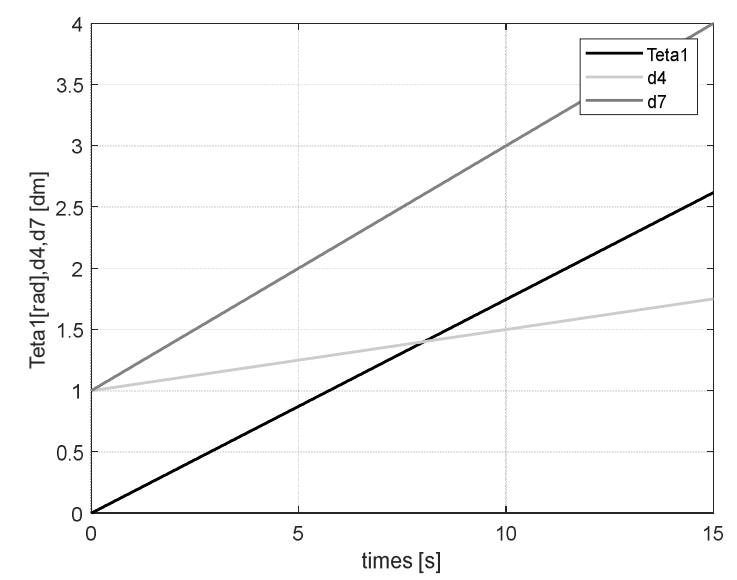

Fig. 5. The curves $\theta_{1}(t), d_{4}(t)$ and $d_{7}(t)$

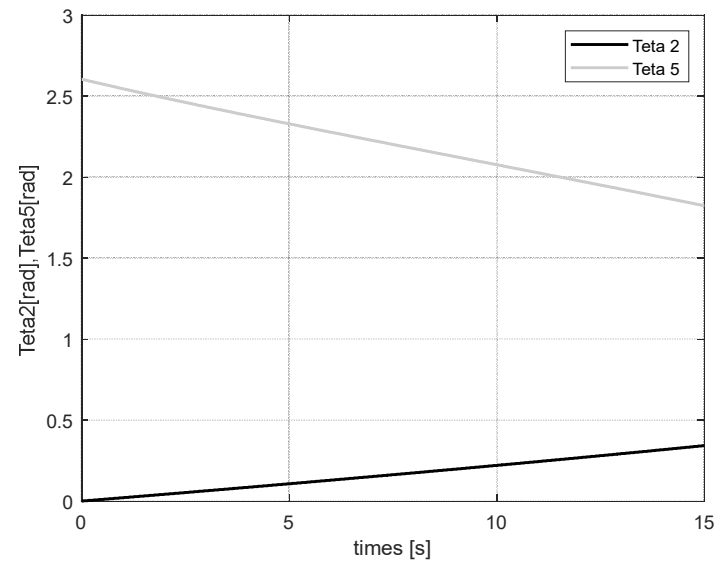

Fig. 6. The curves $\theta_{2}(t)$ and $\theta_{5}(t)$

To validate the inverse kinematic computation, a numerical experiment is carried out. The robot configurations at $t=0 \mathrm{sec}$ and $t=10 \mathrm{sec}$ are shown in Figs. 7 and 8. In 


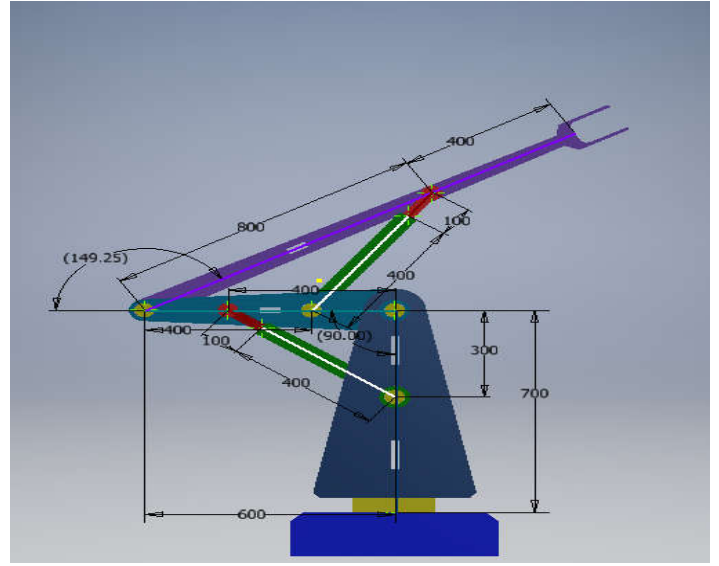

Fig. 7. The robot configuration at $t=0$

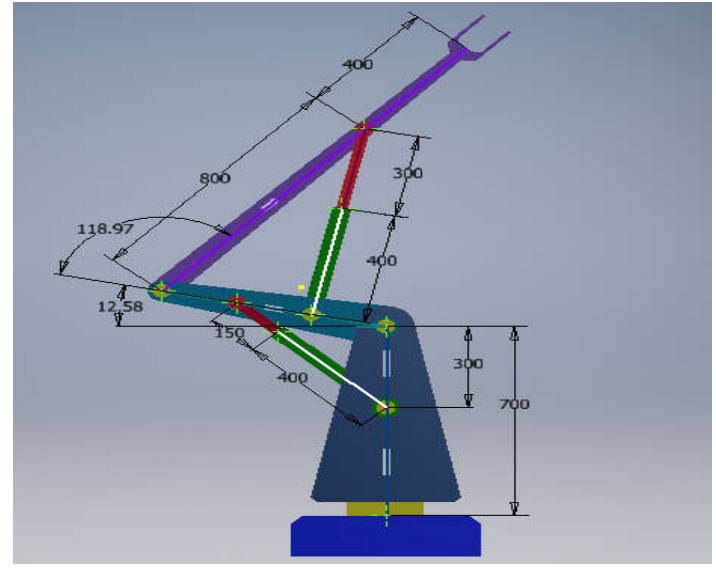

Fig. 8. The robot configuration at $t=10 \mathrm{sec}$

Figs. 7 and 8 , the two revolute joints $\theta_{2}$ and $\theta_{5}$ are driven with two hydraulic cylinders which represent the active joints $d_{4}$ and $d_{7}$. All the parameters of the robot are shown in Figs. 7 and 8 as well.

\section{DYNAMICS}

Let's denote

$\mathbf{s}=\left[\theta_{1} d_{4} d_{7} \theta_{2} \theta_{3} \theta_{5} \theta_{6}\right]^{T}$ is the vector of redundant generalized coordinates;

$\mathbf{q}=\left[\theta_{1} d_{4} d_{7}\right]^{T}$ is the vector of independent generalized coordinates;

$\mathbf{z}=\left[\theta_{2} \theta_{3} \theta_{5} \theta_{6}\right]^{T}$ is the vector of dependent generalized coordinates;

$\mathbf{f}=\left[\begin{array}{llll}f_{1} & f_{2} & f_{3} & f_{4}\end{array}\right]^{T}$ is the vector of constraint equations;

$\Phi_{s}=\frac{\partial \mathbf{f}}{\partial \mathbf{s}}$ is the Jacobian matrix of constraint equations;

$\lambda=\left[\begin{array}{llll}\lambda_{1} & \lambda_{2} & \lambda_{3} & \lambda_{4}\end{array}\right]^{T}$ is the vector of Lagrangian Multipliers;

$\tau=\left[\begin{array}{lllllll}\tau_{1} & F_{4} & F_{7} & 0 & 0 & 0 & 0\end{array}\right]^{T}$ is the vector of applied torque/forces.

By using the Lagrangian formulation, the system of equations of motion including constraint equations for the robot can be written as

$$
\begin{gathered}
\mathbf{M}(\mathbf{s}, t) \ddot{\mathbf{s}}+\mathbf{C}(\mathbf{s}, \dot{\mathbf{s}}, t) \dot{\mathbf{s}}+\mathbf{g}(\mathbf{s}, t)+\Phi_{s}^{T} \lambda=\tau(t), \\
\mathbf{f}(\mathbf{s}, t)=\mathbf{0} .
\end{gathered}
$$

The global mass matrix can be computed as [1]

$$
\mathbf{M}(\mathbf{s}, t)=\sum_{i=1}^{7}\left(m_{i} \mathbf{J}_{T_{i}}^{T} \mathbf{J}_{T_{i}}+\mathbf{J}_{R_{i}}^{T} \mathbf{A}_{i} \mathbf{I}_{i} \mathbf{A}_{i}^{T} \mathbf{J}_{R_{i}}\right),
$$

where $m_{i}$ and $\mathbf{I}_{i}$ are the mass and the inertia of body $i$, respectively; $\mathbf{J}_{T_{i}}$ and $\mathbf{J}_{R_{i}}$ are translational and rotational Jacobian matrices of body $i$, accordingly. 
The angular velocity of the links are calculated as follows:

$$
\begin{gathered}
\omega_{1}=\left[\begin{array}{l}
0 \\
0 \\
\dot{\theta}_{1}
\end{array}\right], \omega_{2}=\left[\begin{array}{l}
\dot{\theta}_{2} \sin \theta_{1} \\
-\dot{\theta}_{2} \cos \theta_{1} \\
\dot{\theta}_{1}
\end{array}\right], \omega_{3}=\omega_{4}=\left[\begin{array}{l}
\dot{\theta}_{3} \sin \theta_{1} \\
-\dot{\theta}_{3} \cos \theta_{1} \\
\dot{\theta}_{1}
\end{array}\right], \\
\omega_{5}=\left[\begin{array}{c}
\sin \theta_{1}\left(\dot{\theta}_{2}+\dot{\theta}_{5}\right) \\
-\cos \theta_{1}\left(\dot{\theta}_{2}+\dot{\theta}_{5}\right) \\
\dot{\theta}_{1}
\end{array}\right], \omega_{6}=\omega_{7}=\left[\begin{array}{c}
\sin \theta_{1}\left(\dot{\theta}_{2}+\dot{\theta}_{6}\right) \\
-\cos \theta_{1}\left(\dot{\theta}_{2}+\dot{\theta}_{6}\right) \\
\dot{\theta}_{1}
\end{array}\right] .
\end{gathered}
$$

The vectors of the mass center of the links are determined as follows:

$$
\begin{aligned}
& \mathbf{r}_{C 1}=\left[\begin{array}{c}
0 \\
0 \\
l_{1} / 2
\end{array}\right], \mathbf{r}_{C 2}=\left[\begin{array}{c}
\frac{1}{2} l_{2} \cos \theta_{1} \cos \theta_{2} \\
\frac{1}{2} l_{2} \sin \theta_{1} \cos \theta_{2} \\
\frac{1}{2} l_{2} \sin \theta_{2}+l_{1}
\end{array}\right], \mathbf{r}_{C 3}=\left[\begin{array}{c}
\frac{1}{2} l_{3} \cos \theta_{1} \cos \theta_{3} \\
\frac{1}{2} l_{3} \sin \theta_{1} \cos \theta_{3} \\
\frac{1}{2} l_{3} \sin \theta_{3}+l_{10}
\end{array}\right], \\
& \mathbf{r}_{C 4}=\left[\begin{array}{l}
\left(l_{3}+d_{4}-\frac{l_{4}}{2}\right) \cos \theta_{1} \cos \theta_{3} \\
\left(l_{3}+d_{4}-\frac{l_{4}}{2}\right) \sin \theta_{1} \cos \theta_{3} \\
\left(l_{3}+d_{4}-\frac{l_{4}}{2}\right) \sin \theta_{3}+l_{10}
\end{array}\right], \mathbf{r}_{C 5}=\left[\begin{array}{c}
\frac{1}{2} l_{5} \cos \theta_{1} \cos \left(\theta_{2}+\theta_{5}\right)+l_{2} \cos \theta_{1} \cos \theta_{2} \\
\frac{1}{2} l_{5} \sin \theta_{1} \cos \left(\theta_{2}+\theta_{5}\right)+l_{2} \sin \theta_{1} \cos \theta_{2} \\
\frac{1}{2} l_{5} \sin \left(\theta_{2}+\theta_{5}\right)+l_{2} \sin \theta_{2}+l_{1}
\end{array}\right], \\
& \mathbf{r}_{C 6}=\left[\begin{array}{c}
\frac{1}{2} l_{6} \cos \theta_{1} \cos \left(\theta_{2}+\theta_{6}\right)+l_{23} \cos \theta_{1} \cos \theta_{2} \\
\frac{1}{2} l_{6} \sin \theta_{1} \cos \left(\theta_{2}+\theta_{6}\right)+l_{23} \sin \theta_{1} \cos \theta_{2} \\
\frac{1}{2} l_{6} \sin \left(\theta_{2}+\theta_{6}\right)+l_{23} \sin \theta_{2}+l_{1}
\end{array}\right] \\
& \mathbf{r}_{C 7}=\left[\begin{array}{c}
\left(l_{6}+d_{7}-\frac{l_{7}}{2}\right) \cos \theta_{1} \cos \left(\theta_{2}+\theta_{6}\right)+l_{23} \cos \theta_{1} \cos \theta_{2} \\
\left(l_{6}+d_{7}-\frac{l_{7}}{2}\right) \sin \theta_{1} \cos \left(\theta_{2}+\theta_{6}\right)+l_{23} \sin \theta_{1} \cos \theta_{2} \\
\left(l_{6}+d_{7}-\frac{l_{7}}{2}\right) \sin \left(\theta_{2}+\theta_{6}\right)+l_{23} \sin \theta_{2}+l_{1}
\end{array}\right] \text {. }
\end{aligned}
$$

The matrix of Coriolis and Centrifugal terms is calculated as [1]

$$
\mathbf{C}(\mathbf{s}, \dot{\mathbf{s}}, t)=\frac{\partial \mathbf{M}(\mathbf{s}, t)}{\partial \mathbf{s}}\left(\mathbf{E}_{n} \otimes \dot{\mathbf{s}}\right)-\frac{1}{2}\left(\frac{\partial \mathbf{M}(\mathbf{s}, t)}{\partial \mathbf{s}}\left(\dot{\mathbf{s}} \otimes \mathbf{E}_{n}\right)\right)^{T},
$$


The potential energy of the system can be calculated as follows

$$
\begin{aligned}
\Pi= & \frac{1}{2} m_{1} g l_{1}+m_{2} g\left(\frac{1}{2} l_{2} \sin \theta_{2}+l_{1}\right)+m_{3} g\left(\frac{1}{2} l_{3} \sin \theta_{3}+l_{10}\right)+m_{4} g\left(l_{10}+\left(l_{3}+d_{4}-\frac{l_{4}}{2}\right) \sin \theta_{3}\right) \\
& +m_{5} g\left(l_{1}+l_{2} \sin \theta_{2}+\frac{1}{2} l_{5} \sin \left(\theta_{2}+\theta_{5}\right)\right)+m_{6} g\left(l_{1}+l_{23} \sin \theta_{2}+\frac{1}{2} l_{6} \sin \left(\theta_{2}+\theta_{6}\right)\right) \\
& +m_{7} g\left(l_{1}+l_{23} \sin \theta_{2}+\left(l_{6}+d_{7}-\frac{l_{7}}{2}\right) \sin \left(\theta_{2}+\theta_{6}\right)\right) .
\end{aligned}
$$

Based on the calculation of the total potential energy of the entire system, the terms related to the gravity effect is calculated as

$$
\mathbf{g}(\mathbf{s}, t)=\left[\frac{\partial \Pi}{\partial s}\right]^{T} .
$$

In order to analyze the dynamic behavior of the robot system, the DAEs $(10,11)$ need to be transformed in a way that the Multipliers are cancelled.

Rewrite $\Phi_{s}=\frac{\partial \mathbf{f}}{\partial \mathbf{s}}$ in the following form

$$
\Phi_{s}=\left[\begin{array}{ll}
\Phi_{q} & \Phi_{z}
\end{array}\right]=\left[\begin{array}{ll}
\frac{\partial \mathbf{f}}{\partial \mathbf{q}} & \frac{\partial \mathbf{f}}{\partial \mathbf{z}}
\end{array}\right]
$$

Let's consider the following expression

$$
\mathbf{R}^{T}=\left[\mathbf{E},-\Phi_{q}^{T}\left(\Phi_{z}^{-1}\right)^{T}\right]
$$

Hence

$$
\mathbf{R}^{T} \Phi_{s}^{T}=\mathbf{0}
$$

Note that

$$
\Phi_{s}^{T}=\left[\begin{array}{cccc}
0 & 0 & 0 & 0 \\
-\cos \theta_{3} & -\sin \theta_{3} & 0 & 0 \\
0 & 0 & -\cos \theta_{6} & -\sin \theta_{6} \\
-l_{22} \sin \theta_{2} & l_{22} \cos \theta_{2} & 0 & 0 \\
l_{3} \sin \theta_{3}+d_{4} \sin \theta_{3} & -l_{3} \cos \theta_{3}-d_{4} \cos \theta_{3} & 0 & 0 \\
0 & 0 & -l_{51} \sin \theta_{5} & l_{51} \cos \theta_{5} \\
0 & 0 & l_{6} \sin \theta_{6}+d_{7} \sin \theta_{6} & -l_{6} \cos \theta_{6}-d_{7} \cos \theta_{6}
\end{array}\right],
$$




$$
\mathbf{R}=\left[\begin{array}{ccc}
1 & 0 & 0 \\
0 & 1 & 0 \\
0 & 0 & 1 \\
0 & -\frac{1}{l_{22} \sin \left(\theta_{2}-\theta_{3}\right)} & 0 \\
0 & -\frac{\cos \left(\theta_{2}-\theta_{3}\right)}{\left(d_{4}+l_{3}\right) \sin \left(\theta_{2}-\theta_{3}\right)} & 0 \\
0 & 0 & \frac{1}{l_{51} \sin \left(\theta_{6}-\theta_{5}\right)} \\
0 & 0 & \frac{\cos \left(\theta_{6}-\theta_{5}\right)}{\left(d_{7}+l_{6}\right) \sin \left(\theta_{6}-\theta_{5}\right)} \\
& \dot{\mathbf{s}}=\mathbf{R} \dot{\mathbf{q}}, \\
\\
\mathbf{s}=\mathbf{R} \ddot{\mathbf{q}}+\dot{\mathbf{R}} \dot{\mathbf{q}} .
\end{array}\right.
$$

Substituting (17), (20), (21) into (10) yields

$$
\overline{\mathbf{M}} \ddot{\mathbf{q}}+\overline{\mathbf{C}} \dot{\mathbf{q}}+\overline{\mathbf{G}} \mathbf{q}=\tau_{\mathbf{q}},
$$

where $\overline{\mathbf{M}}=\mathbf{R}^{T} \mathbf{M}(\mathbf{s}, t) \mathbf{R}, \overline{\mathbf{C}}=\mathbf{R}^{T}(\mathbf{M}(\mathbf{s}, t) \dot{\mathbf{R}}+\mathbf{C}(\mathbf{s}, \dot{\mathbf{s}}, t) \mathbf{R}), \overline{\mathbf{G}}=\mathbf{R}^{T} \mathbf{g}(\mathbf{s}, t)$, and $\tau_{\mathbf{q}}=$ $\mathbf{R}^{T} \tau(t)$.

Eq. (22) is expressed in term of independent generalized coordinates, $\mathbf{q}=\left[\theta_{1} d_{4} d_{7}\right]^{T}$. Notice that all the formulations above are implemented and demonstrated in Maple environment. The following section shows the numeric solutions of the forward and inverse dynamic issues.

\begin{tabular}{|c|c|c|c|c|c|c|c|c|c|c|}
\hline \multirow{2}{*}{ Link } & \multicolumn{3}{|c|}{ Center of gravity } & \multirow{2}{*}{ Mass } & \multicolumn{6}{|c|}{ Inertia } \\
\hline & $x_{C}$ & $y_{C}$ & $z_{C}$ & & $I_{x x}$ & $I_{y y}$ & $I_{z z}$ & $I_{x y}$ & $I_{y z}$ & $I_{z x}$ \\
\hline 1 & 0 & $l_{C 1}$ & 0 & $m_{1}$ & $I_{1 x}$ & $I_{1 y}$ & $I_{1 z}$ & 0 & 0 & 0 \\
\hline 2 & $l_{C 2}$ & 0 & 0 & $m_{2}$ & $I_{2 x}$ & $I_{2 y}$ & $I_{2 z}$ & 0 & 0 & 0 \\
\hline 3 & 0 & 0 & $l_{C 3}$ & $m_{3}$ & $I_{3 x}$ & $I_{3 y}$ & $I_{3 z}$ & 0 & 0 & 0 \\
\hline 4 & 0 & $l_{C 4}$ & 0 & $m_{4}$ & $I_{4 x}$ & $I_{4 y}$ & $I_{4 z}$ & 0 & 0 & 0 \\
\hline 5 & $l_{C 5}$ & 0 & 0 & $m_{5}$ & $I_{5 x}$ & $I_{5 y}$ & $I_{5 z}$ & 0 & 0 & 0 \\
\hline 6 & 0 & 0 & $l_{C 6}$ & $m_{6}$ & $I_{6 x}$ & $I_{6 y}$ & $I_{6 z}$ & 0 & 0 & 0 \\
\hline 7 & 0 & $l_{C 7}$ & 0 & $m_{7}$ & $I_{7 x}$ & $I_{7 y}$ & $I_{7 z}$ & 0 & 0 & 0 \\
\hline
\end{tabular}

\subsection{Forward dynamics simulation}

The dynamical parameters of the robot system are given in Tab. 4

Table 4. The parameters of the robot

$l_{1}=0.7 \mathrm{~m} ; l_{10}=0.4 \mathrm{~m} ; l_{11}=0.3 \mathrm{~m} ; l_{2}=0.6 \mathrm{~m} ; l_{20}=0.2 \mathrm{~m} ; l_{21}=0.4 \mathrm{~m} ; l_{22}=0.4 \mathrm{~m} ; l_{23}=$ $0.2 \mathrm{~m} ; l_{3}=0.4 \mathrm{~m} ; l_{4}=0.4 \mathrm{~m} ; l_{5}=1.2 \mathrm{~m} ; l_{51}=0.8 \mathrm{~m} ; l_{52}=0.4 \mathrm{~m} ; l_{6}=0.4 \mathrm{~m} ; l_{7}=0.4 \mathrm{~m}$. 
We assume that $l_{C 1}=l_{1} / 2=0.35 \mathrm{~m} ; l_{C 2}=l_{2} / 2=0.3 \mathrm{~m} ; l_{C 3}=l_{3} / 2=0.2 \mathrm{~m} ; l_{C 5}=l_{5} / 2$ $=0.6 \mathrm{~m} ; l_{C 6}=l_{6} / 2=0.2 \mathrm{~m} ; l_{C 4}=l_{4} / 2=0.2 \mathrm{~m} ; l_{C 7}=l_{7} / 2=0.2 \mathrm{~m} ;-\frac{5 \pi}{6} \leq \theta_{1} \leq \frac{5 \pi}{6}$; $0.1 \leq d_{4}<l_{4} ; 0.1 \leq d_{7}<l_{7}, m_{1}=80 \mathrm{~kg} ; m_{2}=60 \mathrm{~kg} ; m_{3}=20 \mathrm{~kg} ; m_{4}=10 \mathrm{~kg} ; m_{5}=50 \mathrm{~kg} ;$ $m_{6}=20 \mathrm{~kg} ; m_{7}=10 \mathrm{~kg}$.

$I_{1 x}=m_{1} l_{1}^{2} / 12 ; I_{1 z}=m_{1} l_{1}^{2} / 12 ; I_{2 x}=0 ; I_{2 y}=m_{2} l_{2}^{2} / 12 ; I_{2 z}=m_{2} l_{2}^{2} / 12 ; I_{3 x}=m_{3} l_{3}^{2} / 12 ;$ $I_{3 y}=m_{3} l_{3}^{2} / 12 ; I_{3 z}=0 ; I_{4 x}=m_{4} l_{4}^{2} / 12 ; I_{4 y}=0 ; I_{4 z}=m_{4} l_{4}^{2} / 12 ; I_{5 x}=0 ; I_{5 y}=m_{5} l_{5}^{2} / 12 ; I_{5 z}=$ $m_{5} l_{5}^{2} / 12 ; I_{6 x}=m_{6} l_{6}^{2} / 12 ; I_{6 y}=m_{6} l_{6}^{2} / 12 ; I_{6 z}=0 ; I_{7 x}=m_{7} l_{7}^{2} / 12 ; I_{7 y}=0 ; I_{7 z}=m_{7} l_{7}^{2} / 12$.

The applied torque/forces are given as

$$
\tau_{1}(t)=1.5 \times \sin (2 t), F_{4}(t)=50(20+t), F_{7}(t)=30(20-t) .
$$

Fig. 9 shows the time evolution of $\theta_{1}(t), d_{4}(t)$ and $d_{7}(t)$.

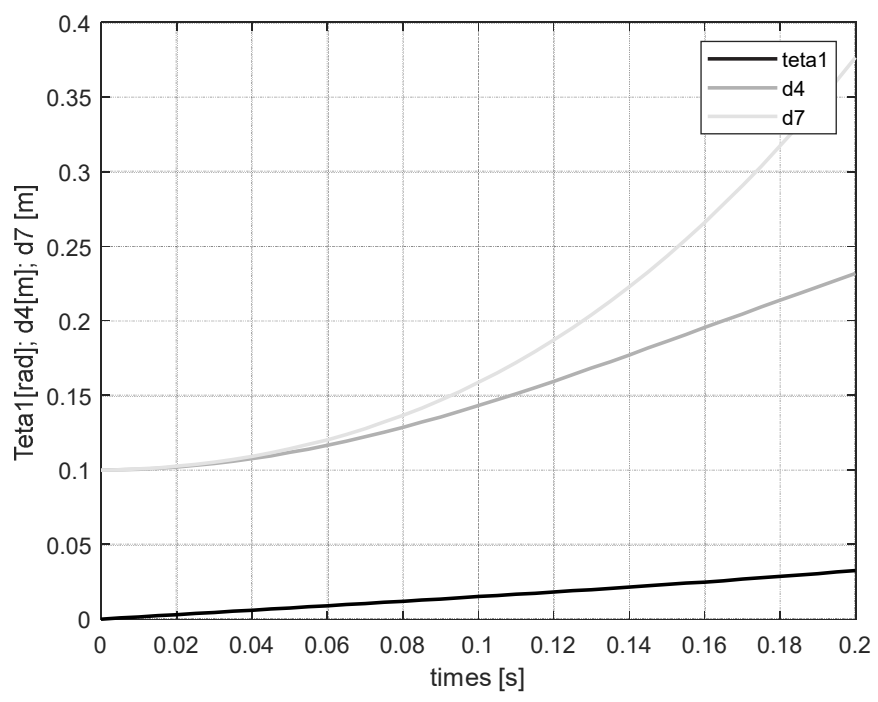

Fig. 9. The time evolution of $\theta_{1}(t), d_{4}(t)$ and $d_{7}(t)$

\subsection{Inverse dynamics simulation}

To demonstrate the inverse dynamic analysis, two cases of simulation are considered. The inputs of the simulation are given as

$$
\theta_{1}(t)=\frac{\pi \times t}{18}, d_{4}(t)=0.1+0.005 \times t, \text { and } d_{7}(t)=0.1+0.02 \times t .
$$

For the first case, the mass of the link $3,4,6$ and 7 equals to zero. In the second case, the mass of the link 3, 4, 6 and 7 are given as $m_{3}=20 \mathrm{~kg}, m_{4}=10 \mathrm{~kg}, m_{6}=20 \mathrm{~kg}$ and $m_{7}=$ $10 \mathrm{~kg}$.

Figs. 10-12 show the results of the inverse dynamic analysis. The "black" curves are the time evolution of the computed torque/forces corresponding to the first case, while the "gray" ones are for the second case. 


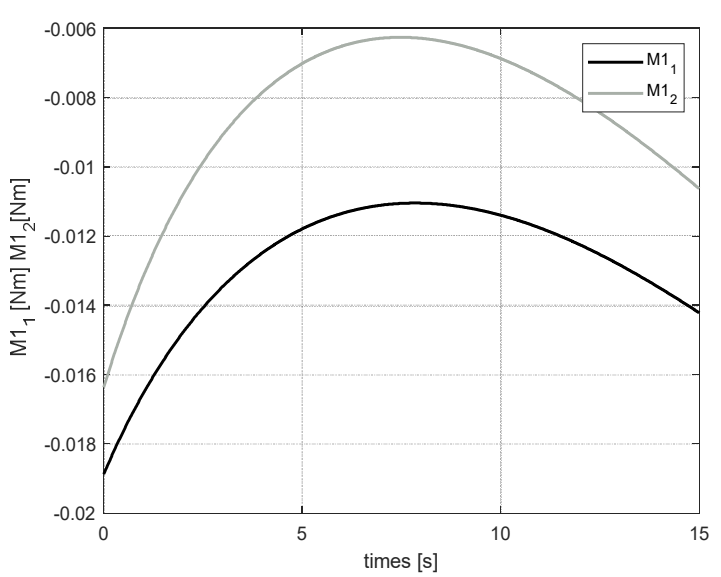

Fig. 10. $\tau_{1}(t)$

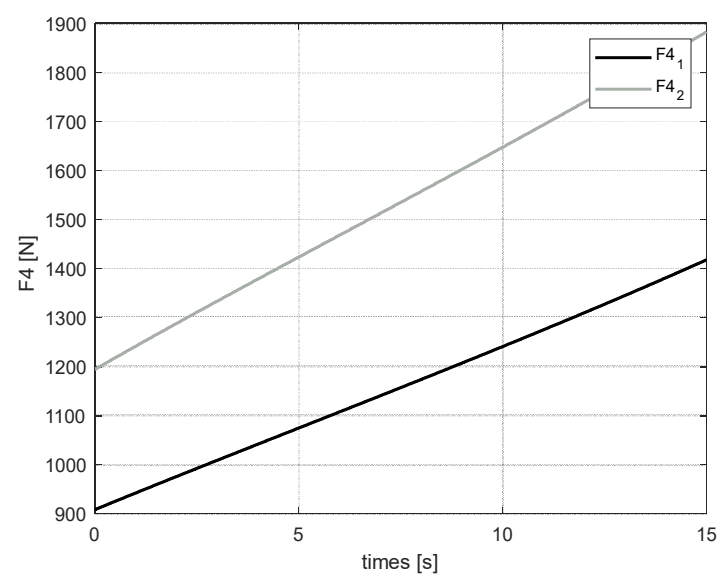

Fig. 11. $F_{4}(t)$

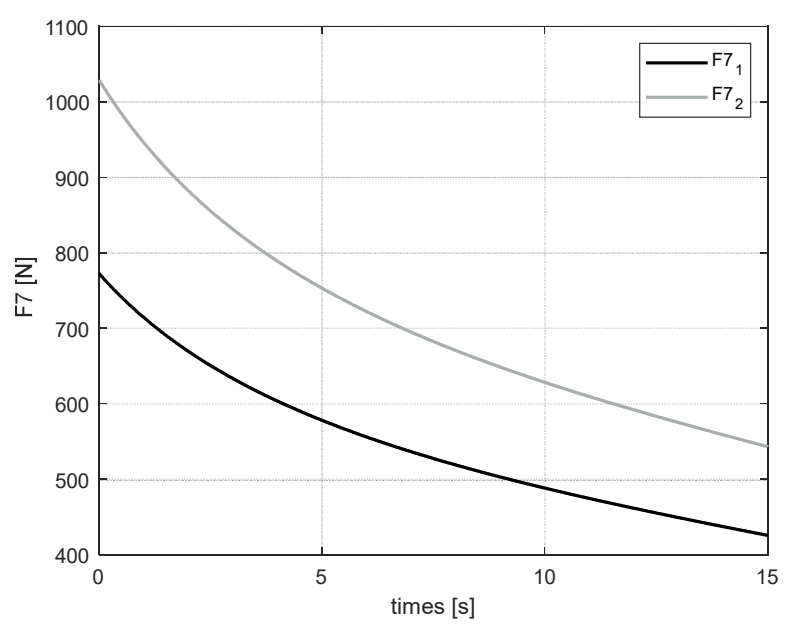

Fig. 12. $F_{7}(t)$

It can be seen that there is a considerable change of the computed torque/forces when considering the mass of the hydraulic cylinders in the dynamic model of the robot system.

\section{CONCLUSION}

The kinematic and dynamic equations for a particular type of robot have been formulated. It has shown that when the mass and inertia of a hydraulic cylinder driving a revolution joint of a robot are considered, such the cylinder and relevant links of the robot constitute a local closed mechanism appended to the main robot architecture. By taking into account this particular feature of the hydraulic robot, the kinematic and dynamic modelling and analysis of the robot are more accurate. This could help to design more efficient and more effective control laws for the arm. 


\section{ACKNOWLEDGMENT}

This research is funded by Vietnam National Foundation for Science and Technology Development (NAFOSTED) under grant number 107.04-2017.09.

\section{REFERENCES}

[1] N. V. Khang and C. A. My. Fundamentals of industrial robots. Vietnam Education Publisher, (2011). (n Vietnamese).

[2] J. J. Craig. Introduction to robotics: Mechanics and control. Pearson Education, Upper Saddle River, NJ, USA, (2005).

[3] J. J. Murray and G. H. Lovell. Dynamic modeling of closed-chain robotic manipulators and implications for trajectory control. IEEE Transactions on Robotics and Automation, 5, (4), (1989), pp. 522-528. https://doi.org/10.1109/70.88066.

[4] V. Mata, S. Provenzano, F. Valero, and J. I. Cuadrado. Serial-robot dynamics algorithms for moderately large numbers of joints. Mechanism and machine Theory, 37, (8), (2002), pp. 739755. https://doi.org/10.1016/s0094-114x(02)00030-7.

[5] H. N. Tran. Inverse kinematic, dynamics and sliding mode control of redundant manipulator. $\mathrm{PhD}$ thesis, Institute of Mechanics, Vietnam Academy of Science and Technology, (2010). (In Vietnamese).

[6] C. A. My. Mechanical design and dynamics modelling of RoPC robot. In Proceedings of International Symposium on Robotics and Mechatronics, Hanoi, Vietnam, (2009). pp. 92-96.

[7] J. P. Merlet. Parallel robots. London Kluwer Academic Publishers, (2000).

[8] O. Ibrahim and W. Khalil. Kinematic and dynamic modeling of the 3-PRS parallel manipulator. In Proceedings of the 12th IFToMM World congress, France, (2007). pp. 1-6.

[9] L. Carbonari, M. Battistelli, M. Callegari, and M. C. Palpacelli. Dynamic modelling of a 3-CPU parallel robot via screw theory. Mechanical Sciences, 4, (1), (2013), pp. 185-197. https://doi.org/10.5194/ms-4-185-2013.

[10] Y. Li and Q. Xu. Kinematics and inverse dynamics analysis for a general 3-PRS spatial parallel mechanism. Robotica, 23, (2), (2005), pp. 219-229. https://doi.org/10.1017/s0263574704000797.

[11] S. Staicu. Matrix modeling of inverse dynamics of spatial and planar parallel robots. Multibody System Dynamics, 27, (2), (2012), pp. 239-265. https://doi.org/10.1007/s11044-011-92818.

[12] W. Khalil and O. Ibrahim. General solution for the dynamic modeling of parallel robots. Journal of Intelligent and Robotic Systems, 49, (1), (2007), pp. 19-37. https://doi.org/10.1007/s10846007-9137-x.

[13] W. H. Yuan and M. S. Tsai. A novel approach for forward dynamic analysis of 3-PRS parallel manipulator with consideration of friction effect. Robotics and Computer-Integrated Manufacturing, 30, (3), (2014), pp. 315-325. https://doi.org/10.1016/j.rcim.2013.10.009.

[14] M. Diaz-Rodriguez, A. Valera, V. Mata, and M. Valles. Model-based control of a 3-DOF parallel robot based on identified relevant parameters. IEEE/ASME Transactions on Mechatronics, 18, (6), (2012), pp. 1737-1744. https://doi.org/10.1109/tmech.2012.2212716.

[15] D. H. Kim, J. Y. Kang, and K. I. Lee. Nonlinear robust control design for a 6 DOF parallel robot. KSME International Journal, 13, (7), (1999), pp. 557-568. https://doi.org/10.1007/BF03186446. 
[16] K. Fu and J. K. Mills. Robust control design for a planar parallel robot. International Journal of Robotics $\mathcal{E}$ Automation, 22, (2), (2007), pp. 139-147. https://doi.org/10.2316/journal.206.2007.2.206-2937.

[17] Y. Li and Q. Xu. Dynamic modeling and robust control of a 3-PRC translational parallel kinematic machine. Robotics and Computer-Integrated Manufacturing, 25, (3), (2009), pp. 630 640. https://doi.org/10.1016/j.rcim.2008.05.006.

[18] H. B. Guo, Y. G. Liu, G. R. Liu, and H. R. Li. Cascade control of a hydraulically driven 6DOF parallel robot manipulator based on a sliding mode. Control Engineering Practice, 16, (9), (2008), pp. 1055-1068. https://doi.org/10.1016/j.conengprac.2007.11.005.

[19] A. Codourey. Dynamic modeling of parallel robots for computed-torque control implementation. The International Journal of Robotics Research, 17, (12), (1998), pp. 1325-1336. https://doi.org/10.1177/027836499801701205.

[20] H. Abdellatif and B. Heimann. Advanced model-based control of a 6-DOF hexapod robot: A case study. IEEE/ASME Transactions On Mechatronics, 15, (2), (2009), pp. 269-279. https://doi.org/10.1109/tmech.2009.2024682.

[21] Q. Li and F. X. Wu. Control performance improvement of a parallel robot via the design for control approach. Mechatronics, 14, (8), (2004), pp. 947-964. https://doi.org/10.1016/j.mechatronics.2004.04.002.

[22] O. Ibrahim and W. Khalil. Inverse and direct dynamic models of hybrid robots. Mechanism and Machine Theory, 45, (4), (2010), pp. 627-640. https://doi.org/10.1016/j.mechmachtheory.2009.11.007.

[23] D. Pisla, A. Szilaghyi, C. Vaida, and N. Plitea. Kinematics and workspace modeling of a new hybrid robot used in minimally invasive surgery. Robotics and Computer-Integrated Manufacturing, 29, (2), (2013), pp. 463-474. https://doi.org/10.1016/j.rcim.2012.09.016.

[24] T. K. Tanev. Kinematics of a hybrid (parallel-serial) robot manipulator. Mechanism and Machine Theory, 35, (9), (2000), pp. 1183-1196. https://doi.org/10.1016/s0094-114x(99)00073-7.

[25] P. Xu, C. F. Cheung, B. Li, L. T. Ho, and J. F. Zhang. Kinematics analysis of a hybrid manipulator for computer controlled ultra-precision freeform polishing. Robotics and ComputerIntegrated Manufacturing, 44, (2017), pp. 44-56. https://doi.org/10.1016/j.rcim.2016.08.003.

[26] Q. Zeng and Y. Fang. Structural synthesis and analysis of serial-parallel hybrid mechanisms with spatial multi-loop kinematic chains. Mechanism and Machine Theory, 49, (2012), pp. 198215. https://doi.org/10.1016/j.mechmachtheory.2011.10.008.

[27] Q. Zeng and K. F. Ehmann. Design of parallel hybrid-loop manipulators with kinematotropic property and deployability. Mechanism and Machine Theory, 71, (2014), pp. 1-26. https://doi.org/10.1016/j.mechmachtheory.2013.08.017.

[28] D. Zhang and Z. Gao. Performance analysis and optimization of a five-degrees-of-freedom compliant hybrid parallel micromanipulator. Robotics and Computer-Integrated Manufacturing, 34, (2015), pp. 20-29. https://doi.org/10.1016/j.rcim.2015.01.002.

[29] C. A. My. Inverse kinematics of a serial-parallel robot used in hot forging process. Vietnam Journal of Mechanics, 38, (2), (2016), pp. 81-88. https://doi.org/10.15625/08667136/38/2/5958.

[30] C. A. My, C. H. Le, M. Packianather, and E. L. J. Bohez. Novel robot arm design and implementation for hot forging press automation. International Journal of Production Research, (2018), pp. 1-15. https://doi.org/10.1080/00207543.2018.1521026.

[31] N. V. Khang, N. P. Dien, N. V. Vinh, and T. H. Nam. Inverse kinematic and dynamic analysis of redundant measuring manipulator BKHN-MCX-04. Vietnam Journal of Mechanics, 32, (1), (2010), pp. 15-26. https://doi.org/10.15625/0866-7136/32/1/313. 